\title{
ZnO/PVP Nanocomposite Spheres with Two Hemispheres
}

Ke Xin Yao and Hua Chun Zeng*

Department of Chemical and Biomolecular Engineering, Faculty of Engineering

National University of Singapore, 10 Kent Ridge Crescent, Singapore 119260

Tel: (65) 6516-2896, Fax: (65) 6779-1936, *Email: chezhc@nus.edu.sg

SI-1 Experimental parameters for the ZnO/PVP samples used in this work:

\begin{tabular}{|l|l|l|l|l|l|}
\hline Sample & $\mathrm{Zn}\left(\mathrm{NO}_{3}\right)_{2} \cdot 6 \mathrm{H}_{2} \mathrm{O}(\mathrm{g})$ & $\mathrm{PVP}(\mathrm{g})$ & $\mathrm{DMF}(\mathrm{mL})$ & Temp $\left({ }^{\circ} \mathrm{C}\right)$ & Time $(\mathrm{h})$ \\
\hline Figure 1b,c & 0.2970 & 0.6 & 40 & 220 & 1 \\
\hline Figure 1d & 0.2970 & 0.6 & 40 & 220 & 20 \\
\hline Figure 1e & 0.0297 & 2.0 & 40 & 220 & 2 \\
\hline Figure 2a & 0.0297 & 0.8 & 40 & 220 & 2 \\
\hline Figure 2b & 0.0445 & 4.0 & 40 & 220 & 2 \\
\hline Figure 2c & 0.0594 & 3.0 & 40 & 220 & 2 \\
\hline Figure 3a,b & 0.2970 & 0.6 & 40 & 220 & 20 \\
\hline Figure 3c & 0.0297 & 1.0 & 40 & 220 & 2 \\
\hline Figure 3d & 0.2970 & 0.6 & 40 & 220 & 2 \\
\hline Figure 3e & 0.0594 & 1.0 & 40 & 220 & 2 \\
\hline Figure 3f & 0.0297 & 4.0 & 40 & 220 & 2 \\
\hline Figure 4a,b & 0.0297 & 2.0 & 40 & 220 & 2 \\
\hline Figure 7a & 0.0445 & 2.0 & 40 & 220 & 2 \\
\hline Figure 7b,c & 0.0297 & 2.0 & 40 & 220 & 3 \\
\hline Figure 8a,b & 0.0297 & $0.8(3.19 \mathrm{eV})$ & 40 & 220 & 2 \\
\cline { 2 - 6 } & 0.0445 & $2.0(3.08 \mathrm{eV})$ & 40 & 220 & 2 \\
\hline SI-2, SI-3 & 0.2970 & 0.6 & 40 & 220 & 2 \\
\hline SI-5 & 0.0445 & 2.0 & 40 & 220 & 2 \\
\hline SI-6 & 0.2970 & 0.2 & 40 & 220 & 2 \\
\hline
\end{tabular}

Note: Zinc nitrate hexahydrate [Zn( $\left.\left(\mathrm{NO}_{3}\right)_{2} \cdot 6 \mathrm{H}_{2} \mathrm{O}\right]$-Reagent grade $98 \%$ (Aldrich); DMF: $N, N$ dimethylformamide [HCON $\left.\left(\mathrm{CH}_{3}\right)_{2}\right]$-ACS reagent 99.8\% (J.T. Baker); and PVP: poly(vinylpyrrolidone) $\left[\left(\mathrm{C}_{6} \mathrm{H}_{9} \mathrm{NO}\right)_{\mathrm{n}}\right]$, average $M_{\mathrm{w}} \sim 55,000$ (Aldrich). 
SI-2 FESEM image for sphere morphology: The boundaries of two hemispheres can be seen clearly in this image (Figure 3d in the main text, also see SI-1).

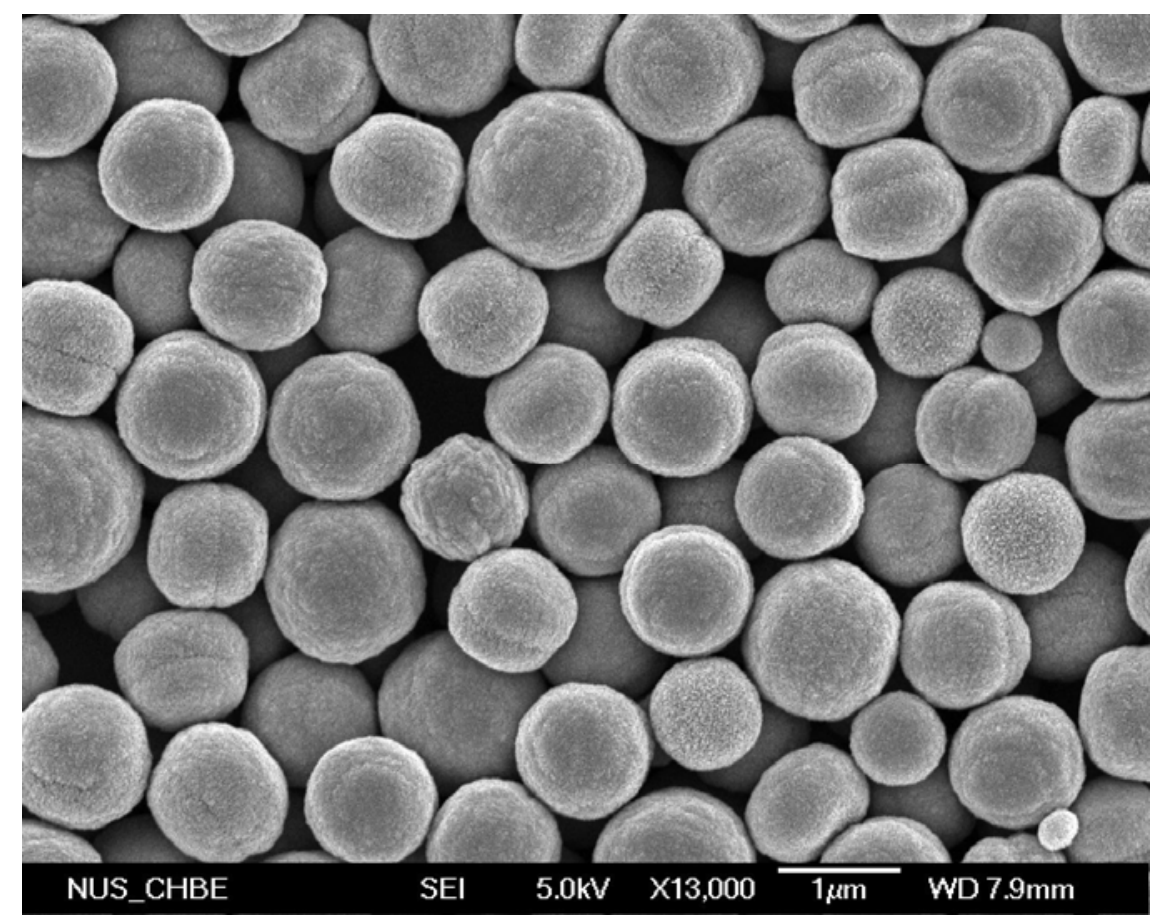

SI-3 Mismatching between two mesocrystal hemispheres: (a) TEM image of a ZnO/PVP sphere viewed along the $c$-axis, and its SAED patterns (b). Two sets of SAED patterns of [0001] zone spots can be found this ZnO/PVP sphere (compared to Figure 3a,b in the main text, and also see SI-1).
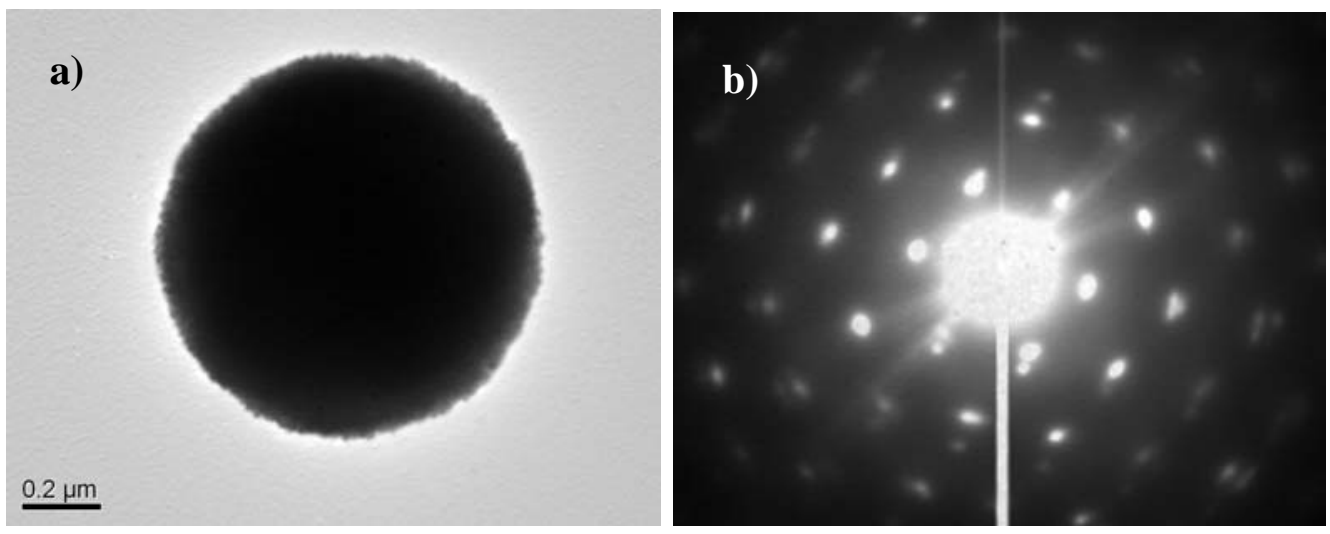
SI-4 EDX analysis for ZnO/PVP composite spheres: Pt signals are from Pt-coating used in FESEM measurement.

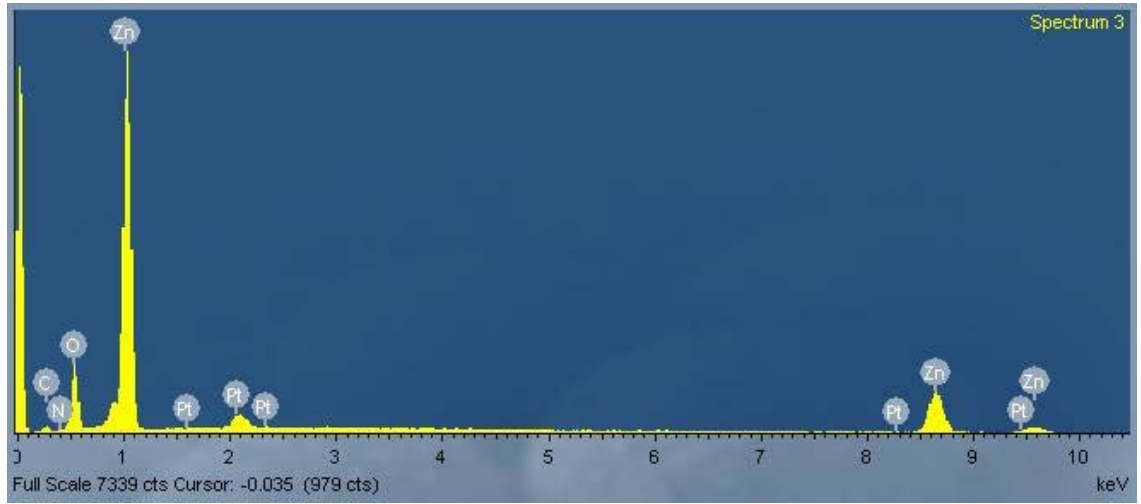

SI-5 Hollow ZnO/PVP spheres (TEM image): A more detailed view on the same sample of hollow spheres shown in Figure 7a of the main text (also see SI-1).

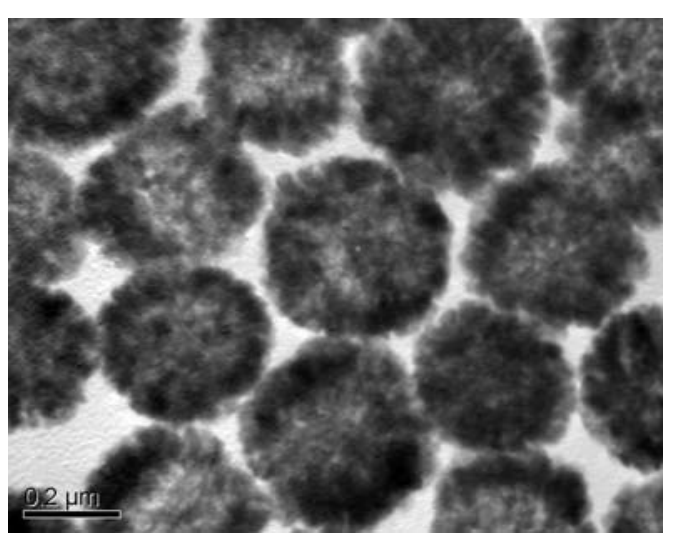


SI-6 TEM ( $a$ and $b$ ) and FESEM (c) images for the bi-crystal sample with low content of PVP in synthesis: See SI-1.

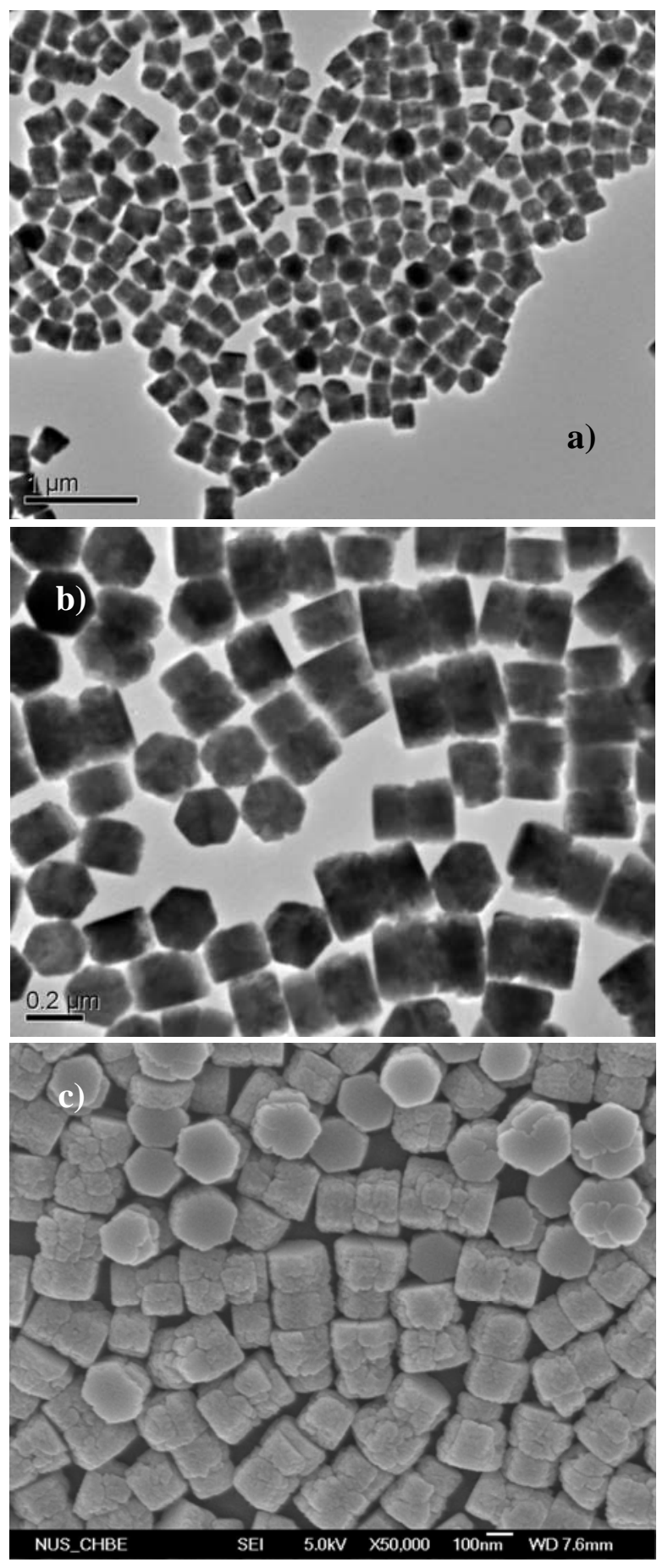

